\title{
Stewart-Treves syndrome: A Rare but Aggressive Complication of Breast Cancer Related-Lymphedema
}

\author{
Pınar Borman $^{1}$ (D), Ayşegül Yaman² (D), Özay Gököz ${ }^{3}$ (D) \\ ${ }^{1}$ Department of Physical Medicine and Rehabilitation, Ankara City Hospital, Ankara, Turkey \\ ${ }^{2}$ Department of Physical Medicine and Rehabilitation, Gülhane Training and Research Hospital, Ankara, Turkey \\ ${ }^{3}$ Department of Pathology, Hacettepe University Faculty of Medicine, Ankara, Turkey
}

\begin{abstract}
Stewart-Treves syndrome (STS) is an angiosarcoma developed on a longstanding lymphedematous extremity. Most of the affected patients have a history of breast cancer that was treated with radical mastectomy. We report a case of STS who has had breast cancer-related lymphedema (BCRL) for 7 years. A 56-year-old woman presented with chronic lymphedema of the right arm. She had modified radical mastectomy for grade 2 invasive ductal breast cancer nine years ago. Physical examination indicated a tender purplish lesion on the medial half of the affected arm. The lesion has spread out quickly with different-sized, scattered, purple-colored lesions on the affected area. A prompt skin biopsy was reported as STS. An immediate arm amputation was performed. But in a few months she presented with new lesions on the anterior thorax and subsequently local recurrence around the scar. Although she had received radiation-therapy, the angiosarcoma spread to pelvic and upper limb area with scattered skin lesions 6 months later. She had several problems during the chemotherapy and radiation-therapy however she reached the survival beyond 20 months. In conclusion Stewart-Treves Syndrome is a rare but aggressive and important complication of BCRL. The awareness of fast-progressing skin lesions and detailed investigation as well as prompt surgical procedures are needed for BCRL patients in order to relatively increase the survival time.
\end{abstract}

Keywords: Breast cancer, lymphedema, lymphangiosarcoma, Stewart-Treves syndrome

Cite this articles as: Borman P, Yaman A, Gököz Ö. Stewart-Treves syndrome: A Rare but Aggressive Complication of Breast Cancer RelatedLymphedema. Eur J Breast Health 29 July 2020. 10.5152/ejbh.2020.5741. [Epub Ahead of Print].

\section{Introduction}

The Stewart-Treves syndrome (STS) is a rare and aggressive angiosarcoma developed from longstanding lymphedematous extremity. Stewart and Treves reported the first lymphangiosarcoma of the upper limb in 6 post-mastectomy patients in 1948 (1). It originates from endothelial cells of lymphatic and blood vessels, but the precise pathomechanism of this phenomenon remains unknown at present $(1,2)$. Diagnosis is made on skin biopsy and imaging studies and the prognosis is poor when radical surgery is not performed (3-5).

We report the case of STS in a 56 years-old woman who underwent modified radical mastectomy for breast carcinoma 9 years earlier and having BCRL for 7 years.

\section{Case Presentation}

A 56-year-old woman presented to our institution with chronic lymphedema of the right arm on October 2016. She had modified radical mastectomy for grade II invasive ductal breast cancer and received chemotherapy, irradiation and hormonotherapy in 2007 and had right arm edema for the last 7 years. Physical examination revealed stage II lymphedema with Stemmer-sign positivity. Inspection indicated a tender purplish lesion $(1.5 \mathrm{~cm} \mathrm{x} 4 \mathrm{~cm}$ ) on the medial half of the affected arm (Figure 1a). She denied any trauma or infection history. No history of comorbidities except hypertension was present. Her routine biochemical test and anticoagulant status were normal. A diagnostic ultrasonography (US) revealed areas of altered echotexture containing subcutaneous multiple hypoechoic masses in the right inner arm with solid and cystic components (Figure 2a, b). Depending on the metastasis suspicion, magnetic-resonance-imaging (MRI) was requested. Up to the MRI result report; hematoma-like yellow color alterations occurred at the edges of the patient's lesion. It has spread out quickly with different sized scattered pink and purple-colored lesions on the affected area (Figure 1b). A prompt skin-biopsy

This case report has been orally presented in 26th World Congress of Lymphology, 25-29 September 2017, Barcelona, Spain 
was performed and demonstrated large, hyperchromatic and atypical neoplastic cells some of which show luminal projections. Low-power view showed extensive infiltration of the dermis by vascular tumor. Pathology also revealed irregular anastomosing vascular channels lined by endothelial cells with different degrees of atypia and mitotic activity, and filling the dermis with cells forming luminal structures, slit-like spaces or small nests. Immunohistochemical staining for CD31 and CD34 was positive and pan-cytokeratin showed no immunoreactivity, indicating angiosarcoma and eliminating cutaneous metastases (Figures 3a-c). MRI indicated dermal diffuse thickness with subcutaneous enhanced-contrasting nodular components, similar to US findings (Figures 4a, b). Depending on these results, STS was diagnosed.

Total abdominal US and chest $\mathrm{x}$-ray were normal. The consultation with oncologist and orthopedic surgeon revealed with immediate forequarter amputation. The surgical margins were found to be clear of tumor with a more than $3 \mathrm{~cm}$-cuff of tumor-free tissue. The clavicular bone biopsy appeared normal without any obvious evidence of local infiltration or metastatic seedling. On her visit 2 months after the operation, the amputation scar was healed clearly (Figure 5) but she had pain and pregabalin-150mg/twice-daily was prescribed for phantom pain.

At 6-weeks control visit, a new lesion on the anterior-thorax was detected (Figure 6). The performed biopsy revealed angiosarcoma again. A wide surgical excision of the tumour was performed on the anterior thorax (Figure 7). Two months later, new lesions around the scar of the
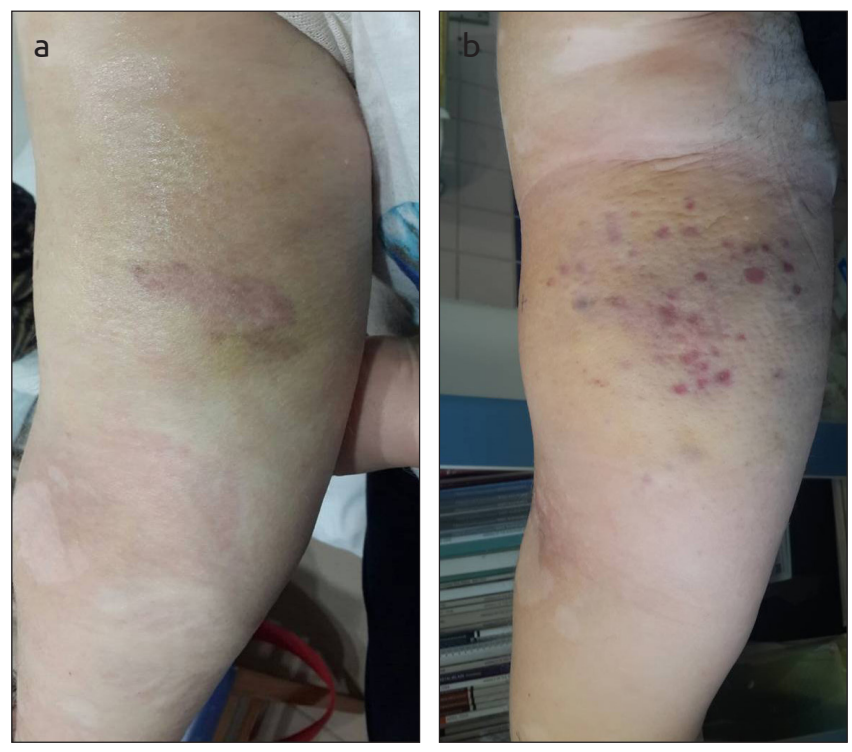

Figure 1. a, b. Purplish lesion which spread out quickly with differentsized, scattered, pink and purple-colored lesions on the right upper extremity

\section{Key Points}

- Stewart-Treves Syndrome is a rare but important complication of BCRL.

- The awareness, early diagnosis, prompt and aggressive therapies and close-monitoring will relatively improve the duration of survival time.

- Diagnostic ultrasonography may be a clinically useful imaging modality to detect the possible malign transformation earlier for BCRL patients with suspicious skin lesions. thoracic mass surgery and on the left axilla were detected (Figures 8a, b). The punch biopsy revealed recurrence of angiosarcoma. Radiationtherapy was performed on the metastatic areas. Three months later she noticed pain and similar lesions on her right hip and upper leg and submitted to oncology and dermatology units (Figures 9). Pelvic bone metastases were revealed on the PET-scan and she received palliative chemotherapy; however, in the third dose of chemotherapy, she presented to the emergency service with fever, dyspnea, cough and breathlessness. She had heavy-pneumonia resistive to multiple therapies and hospitalized in intensive care unit for 3 weeks. After the symptoms and findings were resolved, she was discharged with palliative suggestions. A few weeks later she received radiation-therapy for the pelvic metastatic area and chemotherapy was initiated again. On her final visit, 3 months after chemotherapies, she had severe pain in low back and legs. She was placed on palliative pain control and subsequently she developed urinary tract infection and was lost due to sepsis.
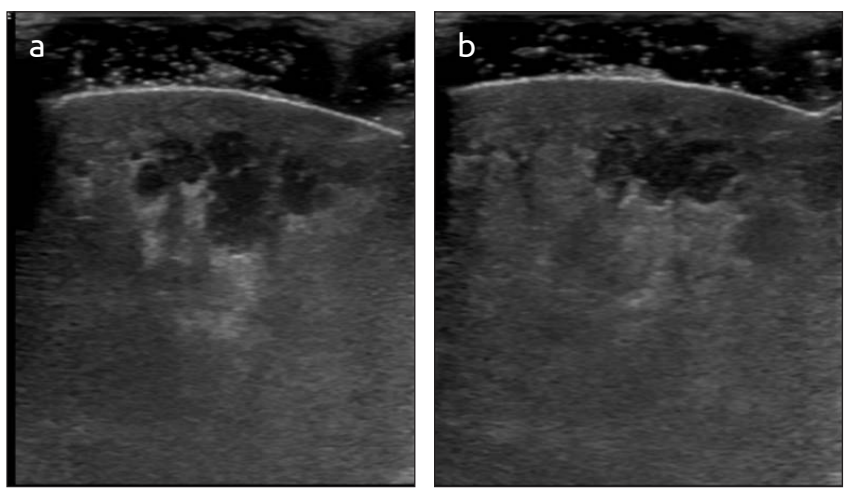

Figure 2. a, b. US revealed multiple masses in the right inner arm with solid and cystic components, subcutaneous, irregular, hypoechoic lesions
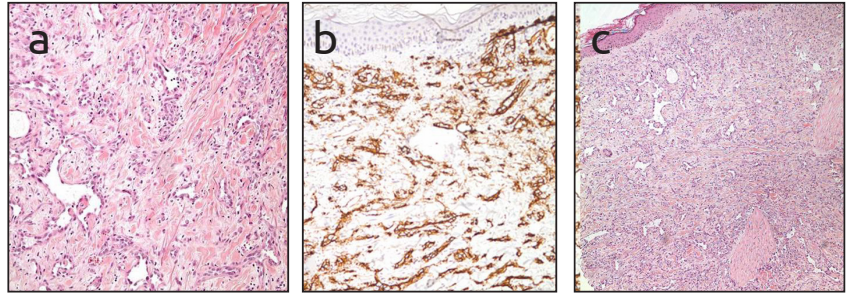

Figure 3. a-c Histological examination revealed irregular anastomosing vascular channels lined by endothelial cells with different degrees of atypia and mitotic activity, and filling the dermis with cells forming luminal structures, slit-like spaces or small nests. Immunohistochemical staining for CD31 and CD34 was positive
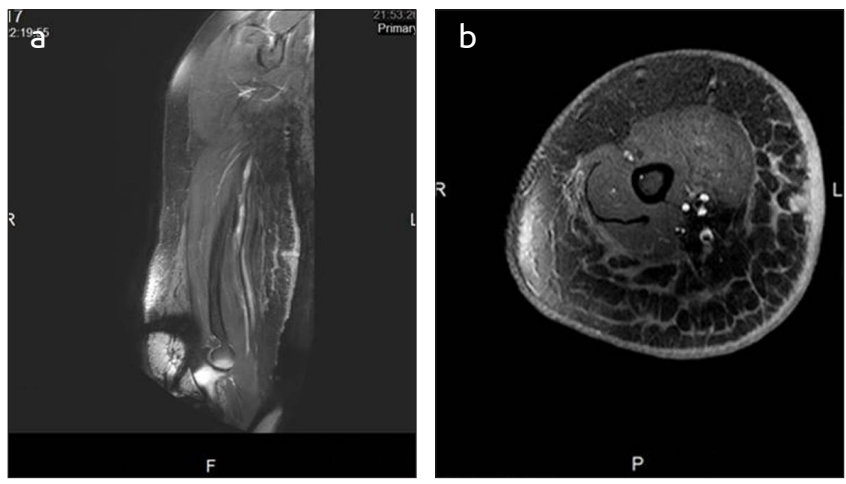

Figure 4. a, b. MRI indicated dermal diffuse thickness with subcutaneous enhanced-contrasting nodular components 


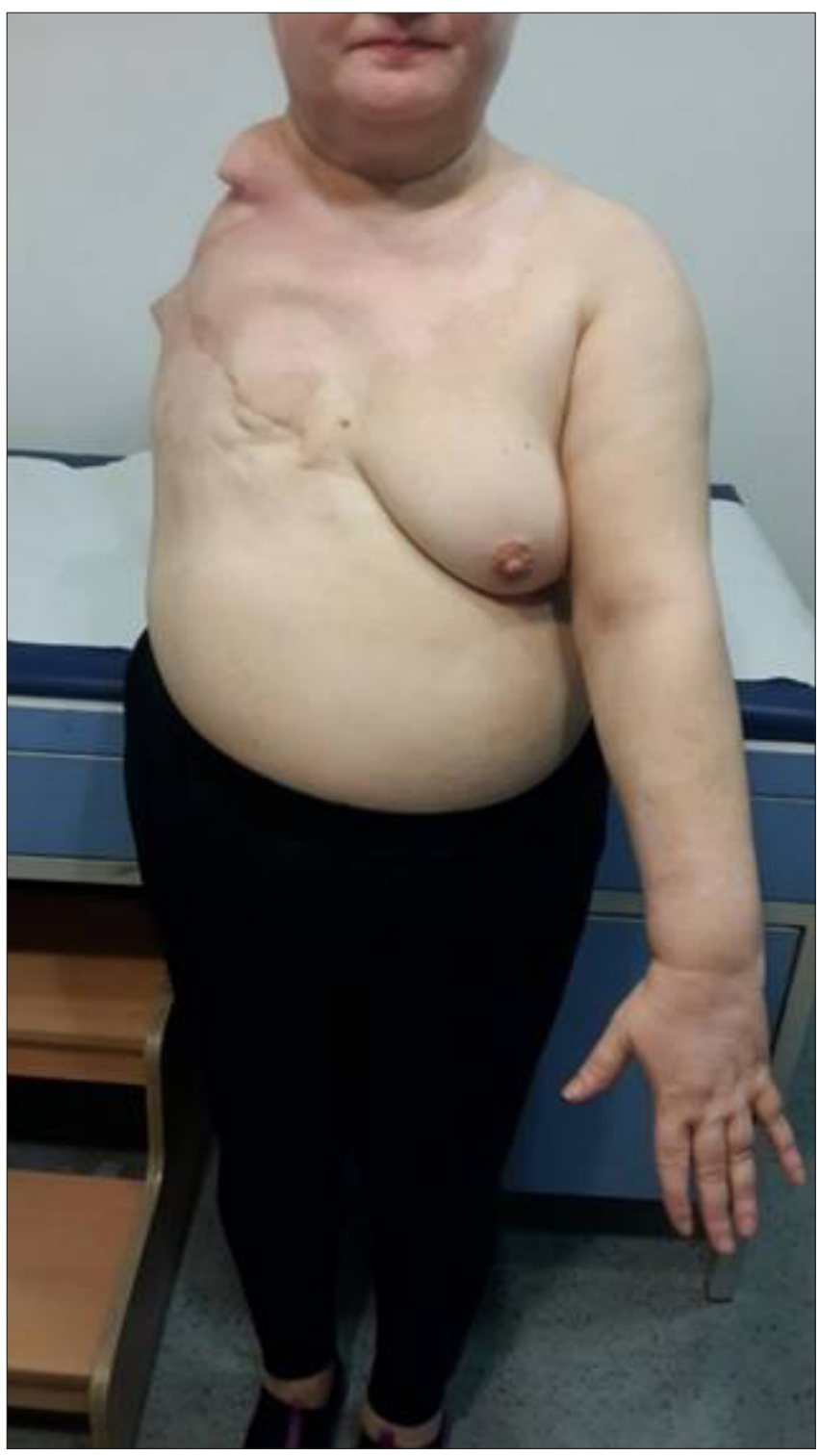

Figure 5. The amputation scar after 2 months

\section{Discussion and Conclusion}

Stewart-Treves syndrome is a rare but deadly angiosarcoma developed on chronic lymphatic obstruction, either primary or secondary (1-10) and estimated at approximately $0.07-0.45 \%$ of patients who survive longer than five years after radical mastectomy $(7,10$, 12). Causative factors comprising radiation-therapy, immunodeficiency and association of STS with cardiovascular conditions have previously defined (11) but the underlying pathophysiology is still debated $(2,13)$. The period between the onset of lymphedema and STS lesions vary between 5-11 years. The tumor is characterized as multiple purplish painless macular lesions which may be dismissed and then develops into a plaque or nodule (3, 7, 13-15). The diagnosis depends on histopathological findings but imagingmodalities can also be helpful $(3,4,15)$. The histopathological findings may vary among cases and commonly seen as irregular and anastomosed endothelial tissue in the most-differentiated areas as well as atypical epithelioid and spindle masses in less-differentiated areas. The immunopathological studies indicate positive staining for endothelial cell markers comprising laminin, antibodies against

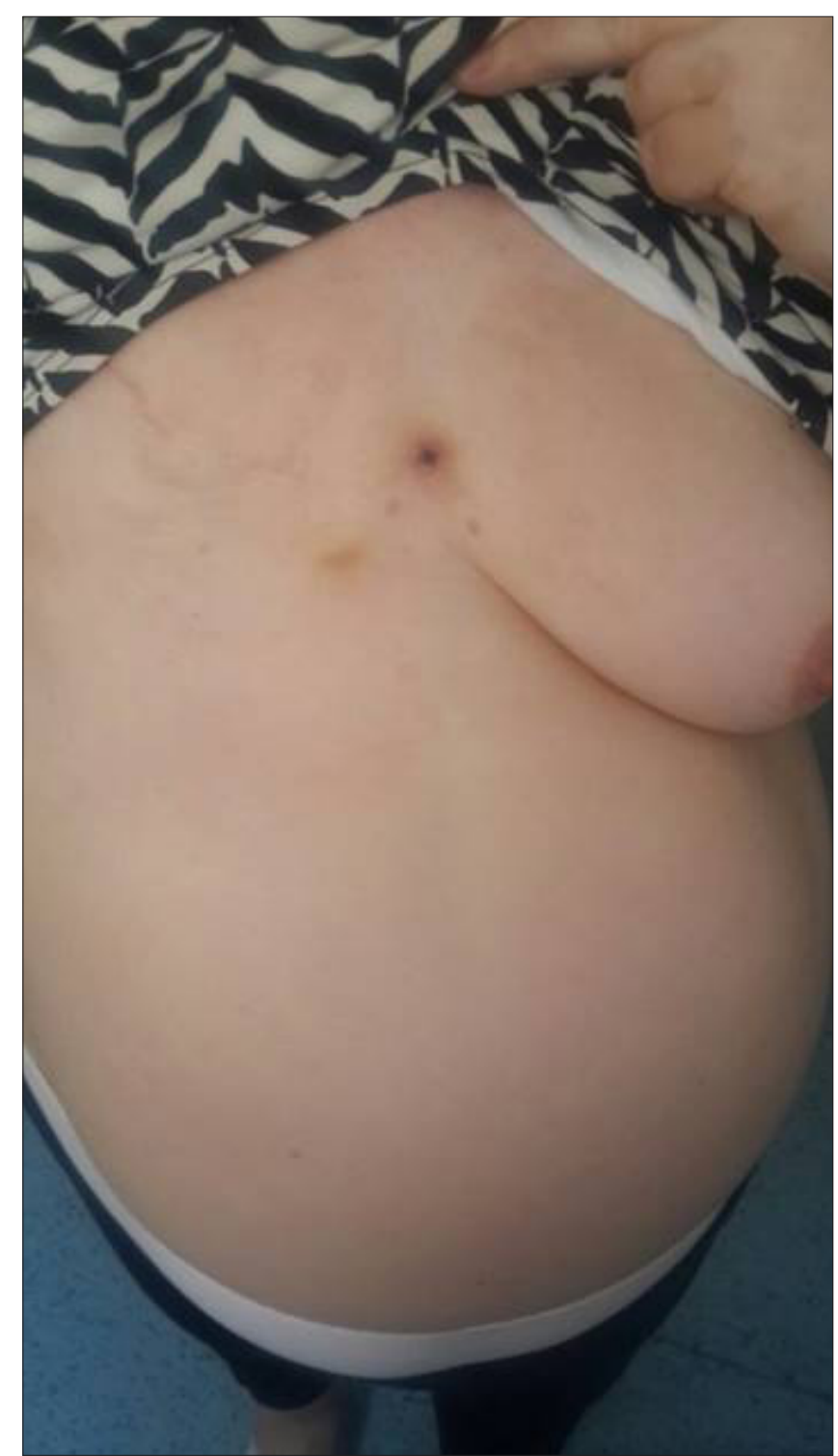

Figure 6. A new lesion on the anterior thorax

CD31 and CD34. Absence of epithelial differentiation markers (EMA, cytokeratin) helps to eliminate cutaneous metastases of breast cancer $(2,4,11,13,15)$.

Our patient had right modified radical mastectomy with resection of 26 axillary-nodes and postoperative radiation 9 years ago, and had lymphedema for more than 7 years. But the age of our patient was relatively young and the survival period was relatively longer to identify the aggressive nature of the tumour and high risk of recurrence different areas and responsiveness to radical therapies. In our case we have performed US and revealed similar lesions with MRI findings. US is widely used in lymphedema patients for differential diagnosis and to monitor effects of treatments in recent years (16). Angiosarcomas have variable features on US examination as well-circumscribed or poorly marginated hypoechoic or hyperechoic masses and US have been previously used to visualize these lesions (17). We suggest diagnostic ultrasonography for evaluation of STS skin lesions and early diagnosis, as a practical, easy and affordable method, but further studies are needed to confirm the diagnostic value and advantages of ultrasonography over MRI or CT in such lesions. 


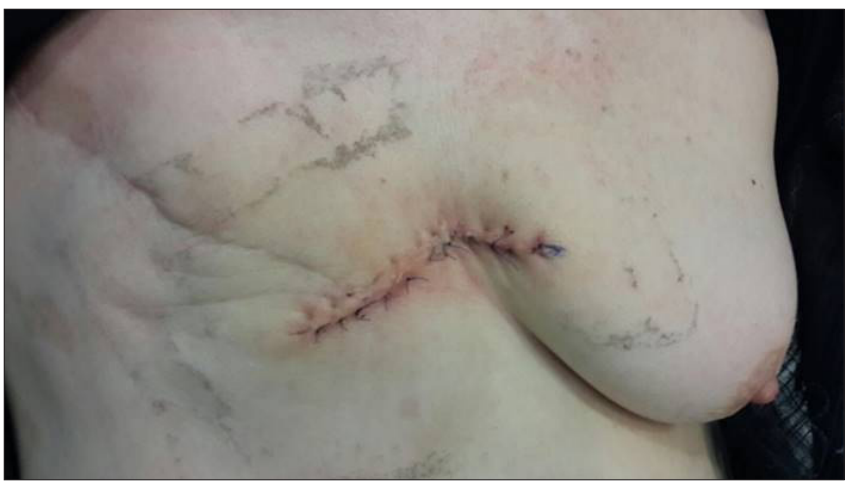

Figure 7. Wide surgical excision of the tumor on the anterior thorax

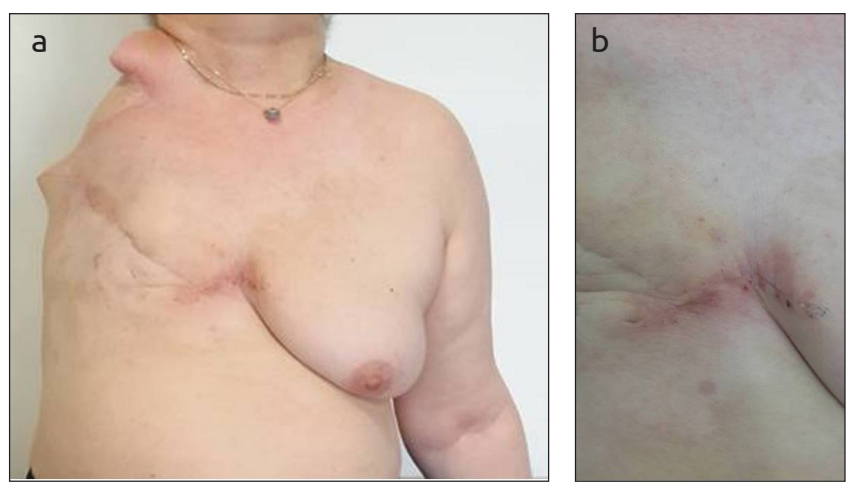

Figure 8. a, b. New lesions around the scar of the thoracic mass surgery, and on the left axilla, 3 months after the second surgery

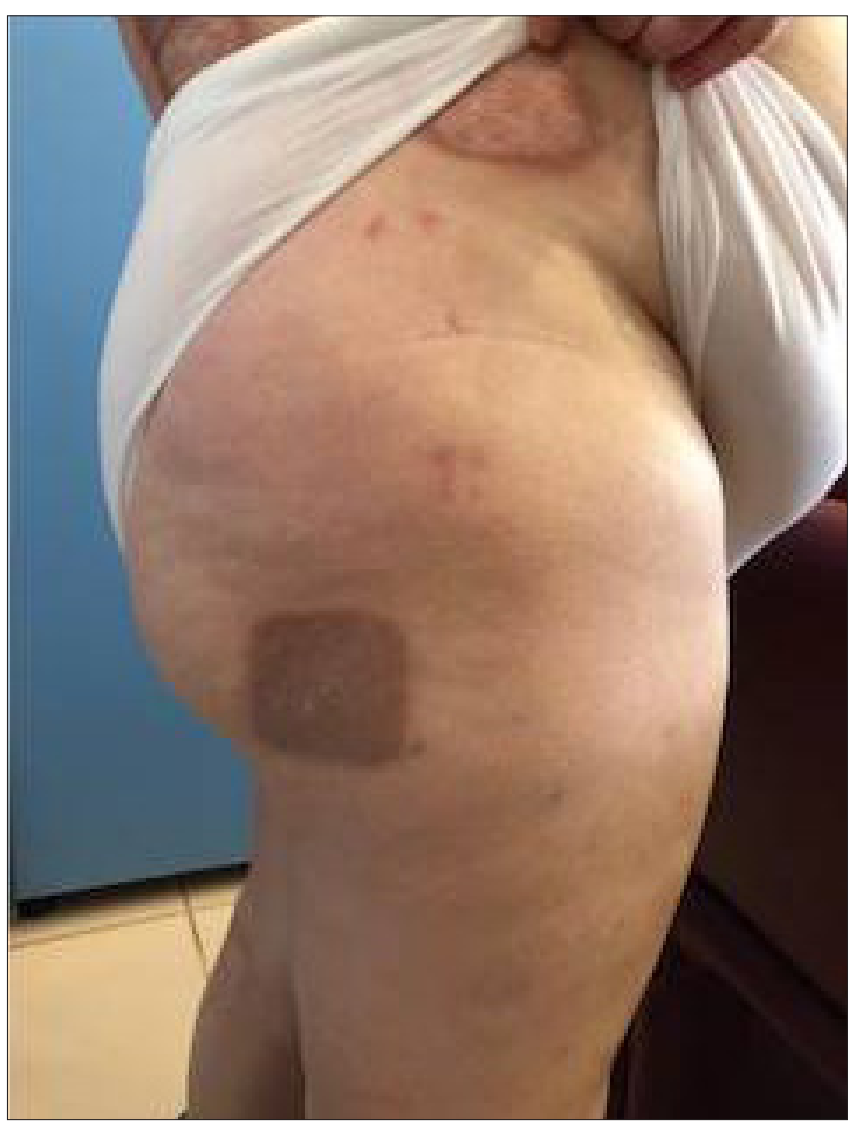

Figure 9. New skin lesions in gluteal area and right upper leg in which punch biopsies were taken
STS has a poor prognosis and due to rarity and high rate of local recurrence and metastatic disease, no standardized therapy is determined (2, 3, $5,7,15)$. As the presentation of the tumor may confuse with traumatic ecchymosis or benign vascular lesions, awareness of this condition is crucial. Our patient has a relatively short duration between the development of lymphedema and presentation of angiosarcoma. After prompt diagnosis, early radical amputation was performed depending on the local extent of the tumor but the tumor was so aggressive that it spread out to the anterior thorax just a few months later and local recurrences and bone metastasis were observed in a few months. Our case also indicates that even amputation may not guaranty being free of local recurrence and recurrences may be common after wide surgical resections. With multi-modal and aggressive management, she managed to reach to survival beyond 20 months.

In conclusion, Stewart-Treves Syndrome is a rare but important complication of BCRL. The awareness, early diagnosis, prompt and aggressive therapies and close monitoring will relatively improve the duration of survival time, as seen in our patient. US may be a clinically useful imaging modality to detect the possible malign transformation earlier for BCRL patients with suspicious skin lesions.

Informed Consent: Written informed consent was obtained from patient who participated in this study.

Peer-review: Externally peer-reviewed.

Author Contributions: Concept - P.B.; Design - P.B.; Resources - A.Y., Ö.G.; Materials - Ö.G.; Literature Search - A.Y.; Writing Manuscript - P.B.; Critical Review - Ö.G.

Conflict of Interest: The authors have no conflicts of interest to declare.

Financial Disclosure: The authors declared that this study has received no financial support.

\section{References}

1. Stewart FW, Treves N. Lymphangiosarcoma in postmastectomy lymphoedema:a report of six cases in elephantiasis chirurgica. Cancer 1948; 1: 64-81. (PMID: 18867440) [Crossref]

2. Sharma A, Schwartz RA: Stewart Treves syndrome: pathogenesis and management. J Am Acad Dermatol 2012; 67: 1342-1348. (PMID: 22682884) [Crossref]

3. Berebichez-Fridman R, Deutsch YE, Joyal TM, Molvera PB, Benedetto PW, Rosenberg AE, Kett DH. Stewart-Treves Syndrome: A case report and review of the literature. Case Rep Oncol 2016; 9: 205-211. (PMID: 27099606) [Crossref]

4. Li B, Wang Z. Stewart-Treves syndrome: magnetic resonance imaging data compared with pathological results from a single center. Oncol Lett 2018; 15: 1113-1118. (PMID: 29391898) [Crossref]

5. Farzaliyev F, Hamacher R, Steinau HU, Bertram S, Podleska LE. Secondary angiosarcoma: A fatal complication of chronic lymphedema. J Surg Oncol 2020; 121: 85-90. (PMID: 31236970) [Crossref]

6. Lee R, Saardi KM, Schwartz RA. Lymphedema related angiogenic tumors and other malignancies. Clin Dermatol 2014; 32: 616-620. (PMID: 25160102) [Crossref]

7. Cui L, Zhang J, Zhang X, Chang H, Qu C, Zhang J, Zhong D. Angiosarcoma (Stewart-Treves syndrome) in postmastectomy patients: report of 10 cases and review of the literature. Int J Clin Exp Pathol 2015; 8: 11108-11115. (PMID: 26617830)

8. Tabareau-Delalande F, de Muret A, Miquelestorena-Standley E, Decouvelaere AV, de Pinieux G. Cutaneous epitheloid clear cells angiosarcoma in a young woman with congenital lymphedema. Case Rep Pathol 2013; 2013: 931973. (PMID: 24078891) [Crossref] 


\section{Eur J Breast Health}

9. Cabral ANF, Rocha RH, Amaral ACVD, Medeiros KB, Nogueira PSE, Diniz LM. Cutaneous angiosarcoma: report of three different and typical cases admitted in a unique dermatology clinic. An Bras Dermatol 2017; 92: 235-238. (PMID: 28538886) [Crossref]

10. Taşdemir A, Karaman H, Ünal D, Mutlu H. Stewart-Treves Syndrome after bilateral mastectomy and radiotherapy for breast carcinoma: Case report. J Breast Health 2015; 11: 92-94. (PMID: 28331699) [Crossref]

11. Felmerer G, Dowlatshahi AS, Stark GB, Földi E, Földi M, Ahls MG, Ströbel P, Aung T. Lymphangiosarcoma: Is Stewart-Treves Syndrome a preventable condition? Lymphat Res Biol 2016; 14: 35-39. (PMID: 26584023) [Crossref]

12. Alan S, Aktas H, Ersoy ÖF, Aktümen A, Erol H. Stewart-Treves syndrome in a woman with mastectomy. J Clin Diagn Res 2016; 10: WD01-2. (PMID: 27042566) [Crossref]

13. Wang LL, Cui LF, Gao Y, Jiang ZC. Clinicopathologic features of Stewart-Treves syndrome. Int J Clin Exp Pathol 2019; 12: 680-688. (PMID: 31933875)
14. Pegas Pereira ES, Siqueira DM, Moraes ET, dos Santos MAS. Stewart Treves Syndrome. An Bras Dermatol 2015; 3(Suppl 1): S229-231. (PMID: 26312725) [Crossref]

15. Mesli SN, Ghouali AK, Benemara F, Taleb FA, Tahraoui H, Abi-Ayad C. Stewart-Treves syndrome involving chronic lymphedema after mastectomy of breast cancer. Case Rep Surg 2017; 2017: 4056459. (PMID: 28280645) [Crossref]

16. Mander A, Venossi S, Menegatti E, Byung-Boong L, Neuhardt D, Maietti E, Gianesini S. Upper limb secondary lymphedema ultrasonographic mapping and characterization. Int Angiol 2019; 38: 334-342. (PMID: 31203598) [Crossref]

17. Taghipour-Zahir S, Sefiddrokh-Sharahjın N, Rahmani K. Primary Breast Angiosarcoma: Pathological and Radiological Diagnosis. Malays J Med Sci 2014; 21: 66-70. (PMID: 25977636) 